\title{
Fragmented nature: consequences for biodiversity
}

\author{
Han Olff ${ }^{\mathrm{a}, *}$, Mark E. Ritchie ${ }^{\mathrm{b}}$ \\ ${ }^{\mathrm{a}}$ Tropical Nature Conservation and Vertebrate Ecology Group, Wageningen University, Bornsesteeg 69, \\ 6708 PD Wageningen, The Netherlands \\ ${ }^{\mathrm{b}}$ Department of Fisheries and Wildlife, Utah State University, Logan, UT 84322-5210, USA
}

\begin{abstract}
We discuss how fragmentation of resources and habitat operate differently on species diversity across spatial scales, ranging from positive effects on local species coexistence to negative effect on intermediate spatial scales, to again positive effects on large spatial and temporal scales. Species with different size and mobility can be regulated by different processes at the same spatial scale, a principle that may contribute to diversity. Differences in species richness between local communities may be differentially regulated at larger spatial scales. This causal connection between local and regional processes has several practical conservation implications. We furthermore show that fractal geometry can be a valuable tool in the separation of the effects of habitat loss (percentage cover), habitat fragmentation (contiguity) and habitat (texture). Especially important may be the notion that the same effective degree of fragmentation can exist at in a very aggregated habitat (one big patch) and a very sparse, random landscape (many small, nearby patches). The geometric behaviour and covariance of these three basic parameters of spatial configuration needs further investigation. The fractal approach is tested using data on heathland habitat configuration and biodiversity in 36 Dutch landscapes of each $9 \mathrm{~km} \times 9 \mathrm{~km}$. Fractal geometry was indeed successful in separating the patterns of habitat loss from habitat fragmentation in a subset of the landscapes, despite covariance across all landscapes. Habitat loss and habitat fragmentation both had a negative effect on diversity of heathland breeding birds, while only habitat loss seemed to affect butterfly species richness. We conclude that fractal geometry seems to be a promising approach for linking population and community processes to landscape spatial structure. (C) 2002 Elsevier Science B.V. All rights reserved.
\end{abstract}

Keywords: Fragmentation; Habitat loss; Biodiversity; Fractal geometry; Scaling

\section{Introduction}

Human alteration of the global environment has triggered the sixth major extinction event in the history of life and has caused wide-spread changes in the abundance and distribution of organisms. Humans

\footnotetext{
* Corresponding author. Present address: Tropical Nature Conservation and Vertebrate Ecology Group, Wageningen University, Bornsesteeg 69, 6708 PD Wageningen, The Netherlands.

Tel.: +31-317-484750; fax: +31-317-484845.

E-mail address: han.olff@staf.ton.wau.nl (H. Olff).
}

have modified biogeochemical cycles (Schlesinger, 1991) have transformed land and enhanced or reduced the mobility of organisms (Chapin et al., 1991). By introducing species into regions that they previously could not reach through natural dispersal, we have triggered biological invasions in some areas with often detrimental effects on the diversity of native species (Elton, 1958, Hobbs and Huenneke, 1992). On the other hand, by fragmenting once continuous natural habitats we have created dispersal barriers for many other species, endangering in their future persistence (Vos and Opdam, 1993). In this paper, we will outline 
some recent ideas on how the spatial fragmentation of food and habitat affects biodiversity. First, we summarise which processes determine diversity across a range of spatial scales, and how fragmentation affects diversity at each of these scales. From this, it will follow that the impact of fragmentation on diversity may be negative or positive, depending on the spatial scale, the temporal scale, and the type of organism involved. After this, we explore various geometric features of habitat fragmentation, discussing the use of fractal geometry for the separation of effect of habitat loss and habitat fragmentation. This method is then applied in a case study on diversity of breeding birds and butterflies in fragmented Dutch heathlands.

\section{Cross-scale determinants of species diversity}

Ecological processes can be classified according to the spatial scale at which they operate. Several current debates on most aspects of diversity regulation suffer from a failure to explicitly consider the spatial scale at which phenomona (e.g. species richness) are evaluated. (Huston, 1999). This may hold especially for the analysis of the consequences of habitat fragmentation for biodiversity, as a specific spatial process. Huston (1999) and Ricklefs and Schluter (1993) distinguish between local and regional determinants of biodiversity. Some local determinants pose limits to the number of species that can persist within some spatial extend, such as competition, predation and parasitism (inter-individual processes). Other local processes however tend to increase this diversity, such as resource differentiation, resource productivity and mutualism. Regional determinants of diversity according to these authors include: (1) differences in age (e.g. of islands); (2) differences in rates of speciation and immigration and (3) differences in extinction.

It indeed seems useful and appropriate to distinguish that different processes govern species composition and diversity at different spatial scales. However, we propose that three instead of two logical aggregation levels of such processes exist: (1) local scale processes; (2) intermediate scale processes and (3) large-scale processes (Fig. 1). This figure shows that the species composition within some spatial extend can always be viewed as a subset of the species composition at some larger spatial extend, with different structuring ecological processes. The few available studies by Holling (1992) and Brown and Maurer (1989) indeed conclude that this may be an appropriate view, mainly from studying patterns in body sizes of species assemblages across spatial scales.

An important consequence of the nested distribution of species (Fig. 1) is that species richness increases towards larger spatial scales. This so-called species-area curve has been extensively investigated by ecologists (see Rosenzweig (1995) for a recent review). The species-area curve often differs strongly between different taxonomic groups (trees, ferns, birds) and between functional groups within these groups (Rosenzweig, 1995). Interestingly, such differences between species-area curves can be used to explore whether different ecological processes structure diversity differently (Fig. 2).

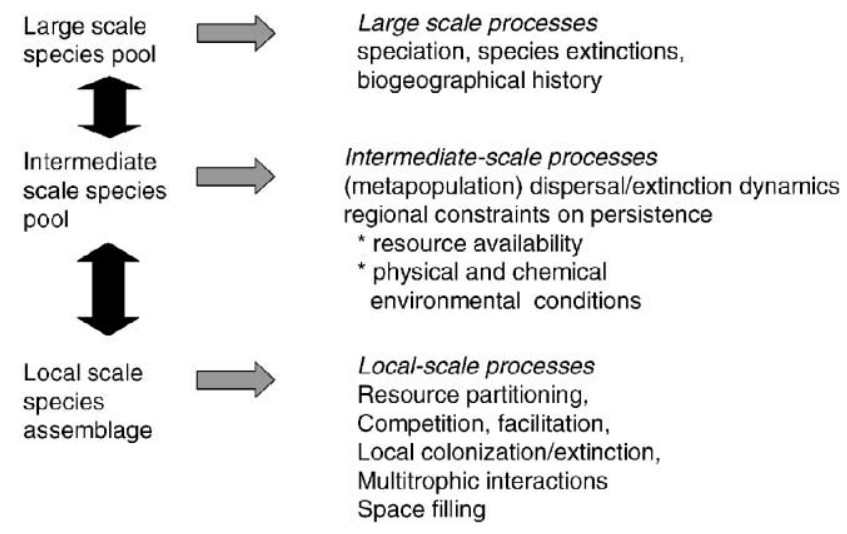

Fig. 1. Cross-scale determinants of biodiversity. 


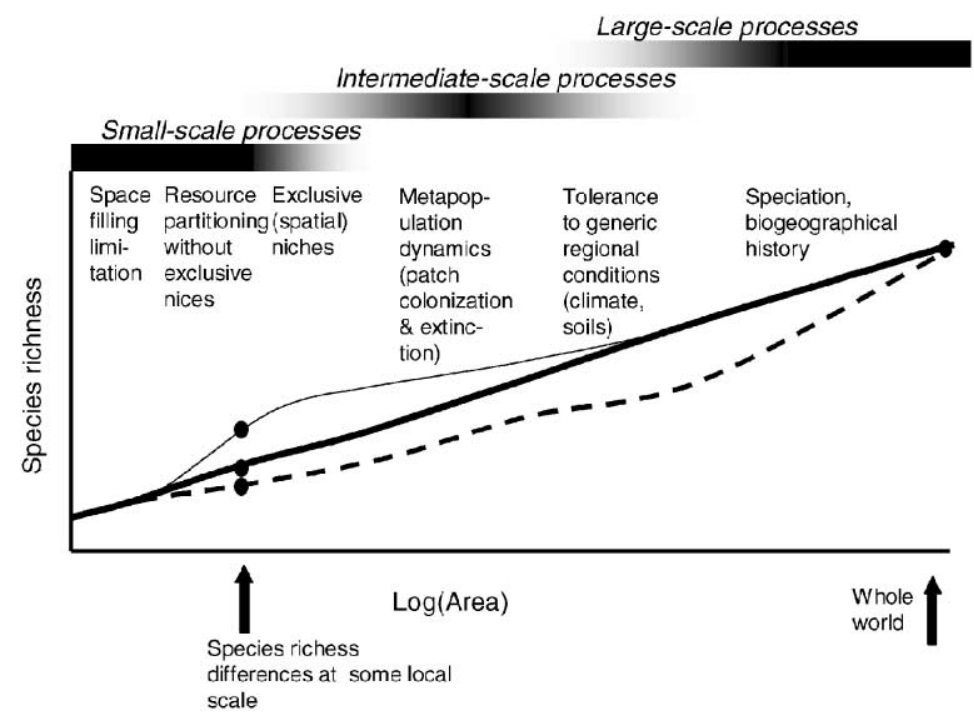

Fig. 2. Hypothetical species-area curves for three different species groups (thin solid line, dashed line and solid bold line) to graph how ecological processes at different spatial scales may structure species diversity different.

Fig. 2 combines two important ecological principles: (1) the species composition at some spatial extend is always a subset of the species composition at a larger spatial extend; and (2) different ecological processes structure species composition at different spatial scales (also see Fig. 1). As a consequence, differences in species richness at some local scale (see arrow in Fig. 2) may be caused at different larger spatial scales. In the case of the patterns depicted in Fig. 2, the species group represented by the thin solid line has less species at a local scale because it has less exclusive niches on local spatial scales. On the contrary, the species group indicated by the dashed line has less species because processes at intermediate spatial scales (as metapopulation dynamics, regional climatic conditions) allow the persistence of less species. A similar comparison could also be done for the same species group, starting on different geographical locations. This could then indicate geographical differences in the relative importance of the processes that structure diversity. We recommend that such analysis should be done more frequently on real datasets to unravel how determinants of diversity change across spatial scales.

Another consequence of the relationships depicted in Fig. 2 is that the number of species present at larger spatial scales affects the species richness of assem- blages at smaller spatial scales. Various authors have indeed observed such a positive correlation of species richness across scales, both for plants and animals (Ricklefs and Schluter, 1993; Pärtel and Zobel, 1996). Care should be taken however to interprete the "species pool" at a larger spatial scale as some passive, random pool of species from which local assemblages are "drawn", as each spatial scale is characterised by its own structuring processes (Figs. 1 and 2).

The effects of fragmentation of resources, food and habitat on species diversity are expected to also vary across spatial scales (Fig. 3). Spatial heterogeneity and fragmentation of resources at small spatial scale has frequently been shown to be associated with higher species richness (Tilman, 1982; Chaneton and Facelli, 1991; Huston, 1994; Knapp et al., 1999), because such differentiation prevents exclusion by a single superior competitor. The opportunities for local coexistence of species can of course be counteracted if the fragmentation results in the conversion or deterioration of natural habitat to unsuitable conditions for any species. At intermediate (regional) spatial scales, effects of fragmentation are generally deterimental, as they negatively affect the balance between the colonisation and extinction rate of suitable habitat patches (Hanski and Simberloff, 1997). At very large spatial scales, fragmentation may again have positive effects on 


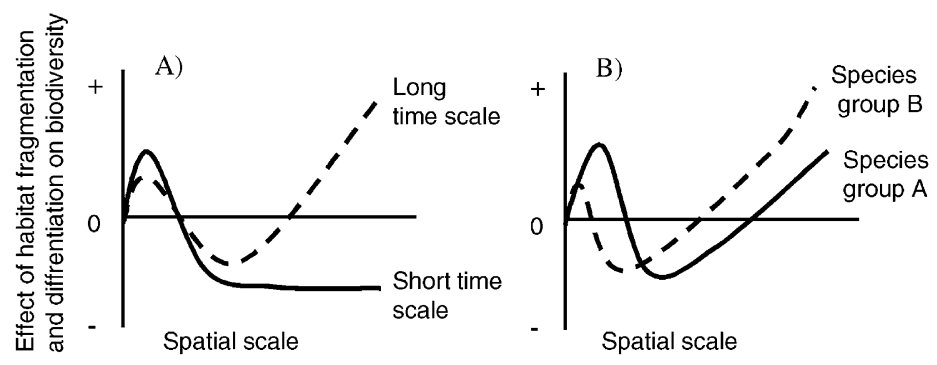

Fig. 3. The effect of fragmentation of resources and habitat across different spatial scales, showing the transition from positive effects on local scales (increased heterogeneity and co-existence), to negative effects on intermediate spatial scales (negative colonisation-extinction balance) to again positive at very large scales (through enhancing speciation), but the latter only at large temporal scales.

diversity, by leading to differential speciation processes of species. The negative impact of biological invasions after human relaxation of large-scale (continental) fragmentation and isolation is a clear example of this. The patterns depicted in Fig. 3 are expected to depend on the nature of the species involved, a pattern that has been studied only little so far. The critical scale at which positive effects of heterogeneity and fragmentation switches to negative impacts is expected to increase with the size and mobiltiy of the species, depending on which spatial area the species integrates over, or "sees" as homogeneous (O’Neill et al., 1989; Turner et al., 1989).

\section{Spatial structure of resources, food and habitat: geometric constraints}

Before discussing the consequences of fragmentation at different spatial scales, we first like to explore its geometrical properties a bit further. The general term fragmentation is an only loosely defined concept and consists of several basic components. Fragmentation in an ecological context is just one aspect of the spatial distribution of some feature (resources, food, habitat). Where most studies have been conducted on the effect of habitat fragmentation (MacArthur and Wilson, 1967; Hanski and Simberloff, 1997), less emphasis has been given to fragmentation of food and resources within habitats (Ritchie and Olff, 1999).

The equilibrium theory of island biogeography as formulated in the 1960s by MacArthur and Wilson (1967), and studies that drew conservation implications from it (Wilson and Willis, 1975) took a simple Euclidean approach to the problem. Smaller patches were considered to be more fragmented than larger patches, single patches were less fragmented than subdivided patches of the same total area, and groups of patches that were closer together were considered less fragmented than further spaced patches.

However, it is increasingly recognised that natural patterns often show very irregular patterns that can only be very roughly characterised by methods of euclidean geometry (Mandelbrot, 1987). A classic example is the question how long a specific coastline (of e.g. Britain, or Norway) is, a question to which the answer is of course relevant for any coastal bird that uses it as a breeding habitat. Surprisingly at first, there is no single answer to this apparently simple question. Instead, the answer depends on the length of the ruler with which we measure this length. If we define the length as the product of the length of the ruler and the number of times we need to flip it over to measure the coastline, we get an ever longer length when using a shorter ruler. When the log of the length of the ruler is plotted against the log of the measured length with that ruler, we usually get a straight line. This indicates that coastlines as a natural shape can not be characterised by integer dimensions in euclidean geometry, but by fractions of a dimension or a "fractal dimension" in so-called fractal geometry (Mandelbrot, 1987). Shapes or patterns that follow a fractal geometry often are scale invariant, meaning that the shape characteristics are independent of the scale at which we look at the shape. Said otherwise, when we enlarge parts of the shape, it will look approximately the same as the entire shape. Not only geological features show such self-similarity, it is also frequently observed in the movement and shape of organisms. This self-similarity of geological and biological shapes is probably 
caused by a small set of physical, chemical and biological processes. A general feature of such processes that generate self-similar, or fractal shapes is that small, local effects may magnify towards larger spatial scales according to a geometric rule. For example, simple local branching rules can lead to self-similar trees and ferns, simple local erosion processes can lead to self-similar mountain ranges, and simple flow principles can lead to fractal river systems. Because of these simple rules, habitat, food and resources for organisms often are found to be statistically self-similar across ecologically relevant ranges of scales (3-4 orders of magnitude) (Milne, 1991, 1992; Milne et al., 1992).

Fractal geometry has proven to be a useful tool in the quantification of spatial patterns in ecology (Johnson et al., 1995) and for a causal analysis of species interactions in spatially structured habitats (Ritchie and Olff, 1999). Fractal geometry is especially important because it provides a tool for describing apparently complex patterns in only a few parameters, which can be used to gain insight in underlying processes. Also, we need to be aware that apparently simple questions on the shape of fractal shapes may have complex answers (see previous coastline example). Fig. 4 illustrates such an apparently simple question. For any habitat that can be viewed as a binary map (present or absent in a cell) we can ask what the percentage cover of that habitat is in the landscape-a critical question of course for ecologists interested in the persistence of a species that depends on that specific habitat. Fig. 4 shows a simple selfsimilar fractal, a so-called inverted Sierpinski carpet. The deterministic form of such a fractal is characterised by an initiator (Fig. 4A) and a generator (Fig. 4B) (Mandelbrot, 1987). In this case, the selfsimilarity of the shape consists of the fact that every white cell in Fig. 4B is similar to the whole shape. The second generation of this fractal (Fig. 4C) is thus obtained by replacing every white cell in Fig. 4B by the whole shape of Fig. 4B. Similar, the third generation pre-fractal is obtained by replacing every white cell in Fig. 4C by a down-scaled version of the shape Fig. 4B. This process can be repeated endlessly, leading to ever increasing detail. The location of the "black" cell in Fig. 4B is not necessarily fixed, when we move it around at random at each use of the generator, we get a so-called stochastic fractal shape
(Fig. 4D-G). Towards the later generations, these stochastic fractals already show that a very simple self-similarity rule leads to apparently complex spatial patterns that look similar to patterns that we observe in the real world (e.g. in the distributions of vegetation types, species or soil types). Most interestingly, there is for such a fractal shape no unique answer to the question what the percentage cover is of the "black" cells-it depends on the pre-fractal generation (Fig. 4H), and thus on the spatial resolution at which we look at the shape. Also, the sizes of the largest and smallest patch are not fixed, as they depend on the extend and resolution at which we view the shape.

Fig. 5A shows an even more realistic landscape, simulated according to a very simple local rule with a stochastic component. The algorithm used for simulating this landscape started with a "black" lattice of $120 \times 120$ cells, of which $1 \%$ were turned white, at random positions, Then, we moved a $3 \times 3$ cell window over the lattice, and turned the central cell in this window white depending on a calculated probability of conversion. This conversion probability depended linearly on the proportion of cells in the $9 \times 9$ window that were already white. Such an algorithm is an example of a simple local rule, that may mimic processes as land-use spread (higher rate of conversion of natural habitat at the edge of larger pieces of cultivated land), but also may mimic the spread of a disease (higher virulence around infected locations). Various methods exist to calculate the fractal features of the such an image, of which we adopted the massfractal dimension approach. For this, we center a window of some length on a focal "occupied"'(black in this case) pixel, and count how many cell are filled inside that window. Then, the count is repeated for larger and larger windows. This process is then repeated for every pixel, resulting in many overlapping windows. The log of the average count of at each window length is then graphed against the log of the window length. The straight line produced in this case (Fig. 5B) shows that the pattern is a fractal, which is characterised by the fractal dimension (slope of the line) and the lacunarity (intercept of the line). Also, the proportional fill can be calculated for the shape, but it should be reminded that this is partly dependent on the resolution of the "map" (Fig. 4), so it can only be compared between maps with the same resolution. 
Deterministic inverse Sierpinski carpet

A) Initiator

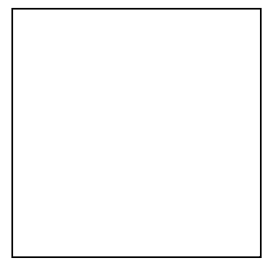

D) Initiator

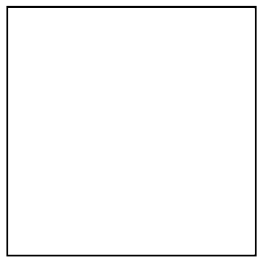

B) Generator (1st prefractal)

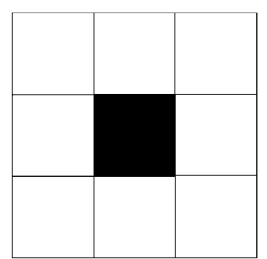

C) $2^{\text {nd }}$ generation prefractal

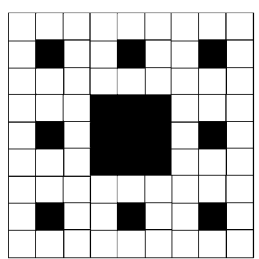

Random inverse Sierpinski carpet

E) Generator (1st prefractal)

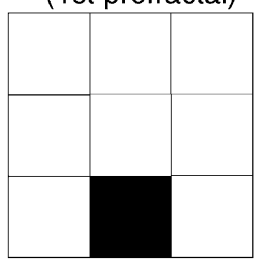

F) $2^{\text {nd }}$ generation prefractal

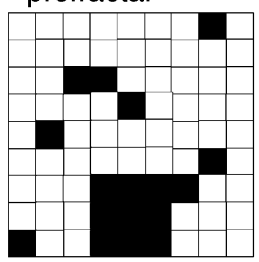

D) $3^{\text {rd }}$ generation prefractal

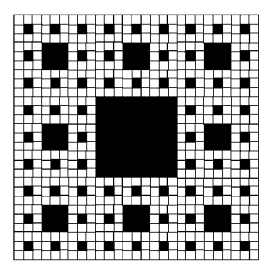

H) Effect of level of detail on proportional fill

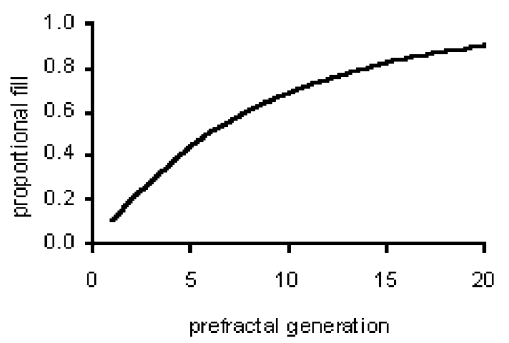

Fig. 4. Illustration of the initiator (A and D) and generator (B and E) of a deterministic (A-D) and stochastic (D-G) fractal, the inverted Sierpinski carpet, with the effect of level of detail (pre-fractal generation) on the proportional fill of the black cells.

A)

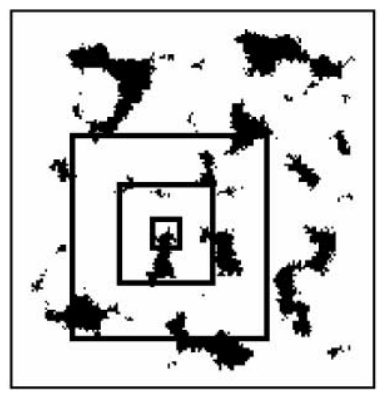

B)

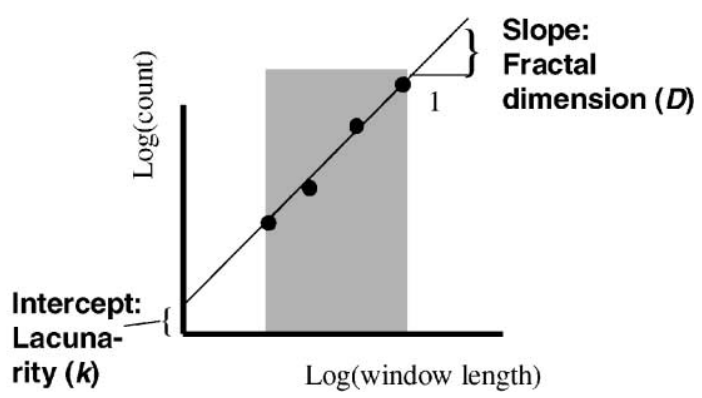

Fig. 5. Calculation of the fractal dimension and lacunarity for a simulated landscape. The fractal dimension is first calculated for each pixel as the slope of the regression of window length around an occupied pixel vs. the number of filled cells in that window. The slope for all the occupied pixels is than averaged to obtain the fractal dimension of the whole landscape. 

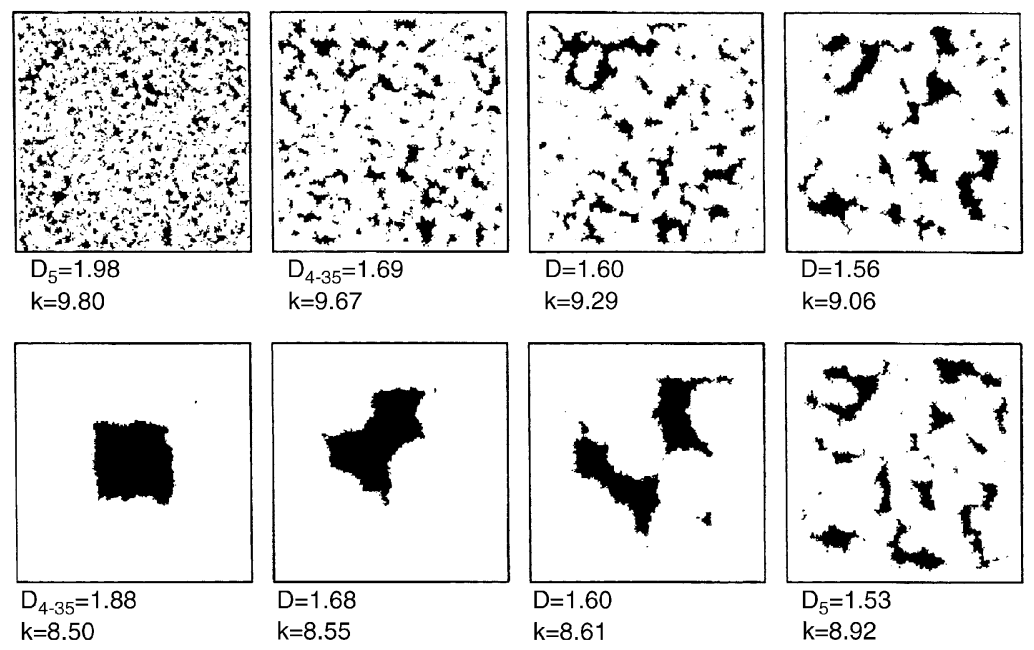

Fig. 6. Simulated landscapes with the same proportional fill (around 10\%) but different combinations of fractal dimension $(D)$ and lacunarity (k). See the text on Fig. 5 for the simulation algorithm.

The fractal dimension calculated this way indicates the contiguity or connectivity of the habitat. The lacunarity reflects the "texture" of the landscape, indicating how much habitat is found in the direct vicinity of existing habitat. Fig. 6 illustrates the usefulness of fractal geometry in differentiating between (simulated) landscapes with different topography and texture. Interestingly, the fractal dimension can be high both when most habitat is aggregated in a one or a few large patches, and when most patches are very small but close together. Both states have in common that the contiguity is high, so the isolation of habitat is relatively low. The difference between the two spatial configurations is captured by the lacunarity.

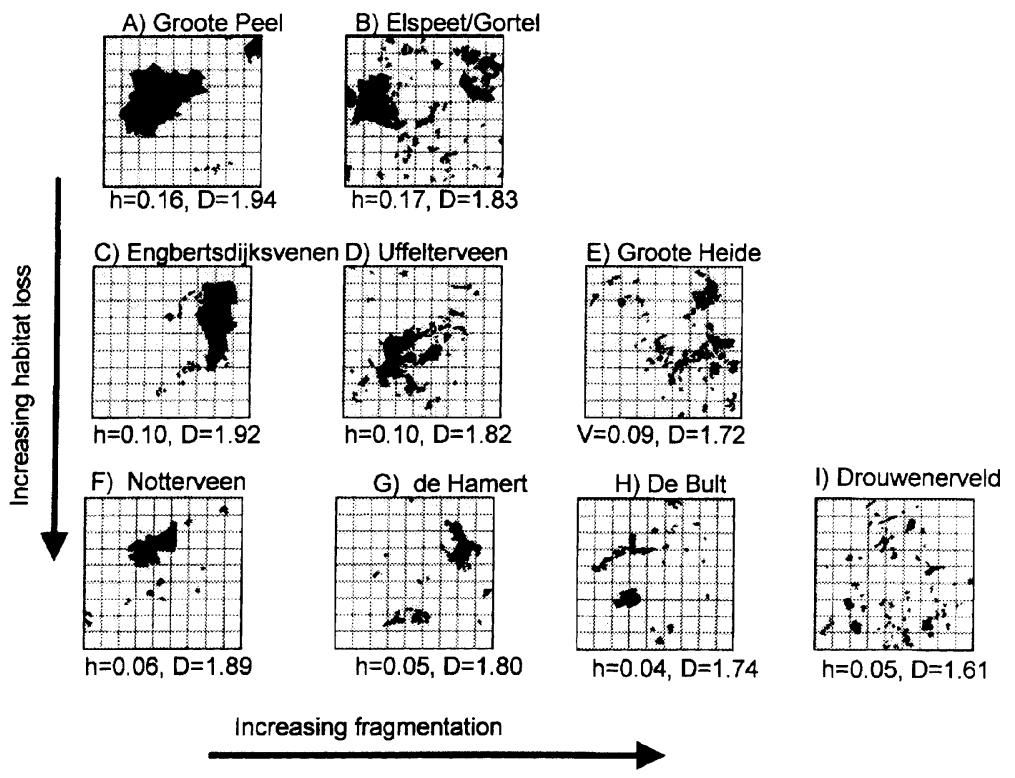

Fig. 7. Simulated landscapes with the same proportional fill (around 10\%) but different combinations of fractal dimension $(D)$ and proportional fill $(h)$. See the text on Fig. 5 for the simulation algorithm. 


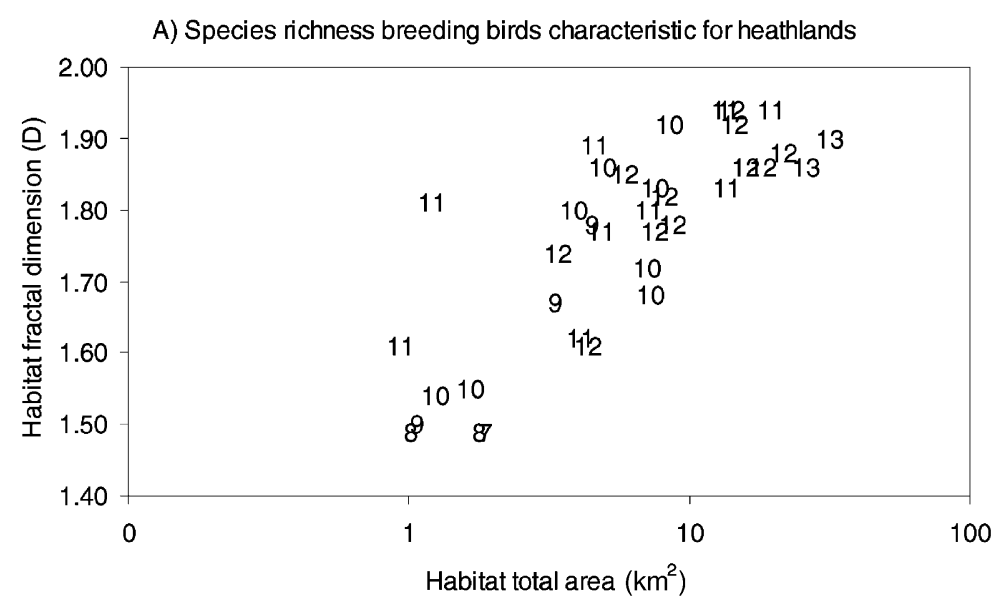

B) Butterfly species richness (heathland species)

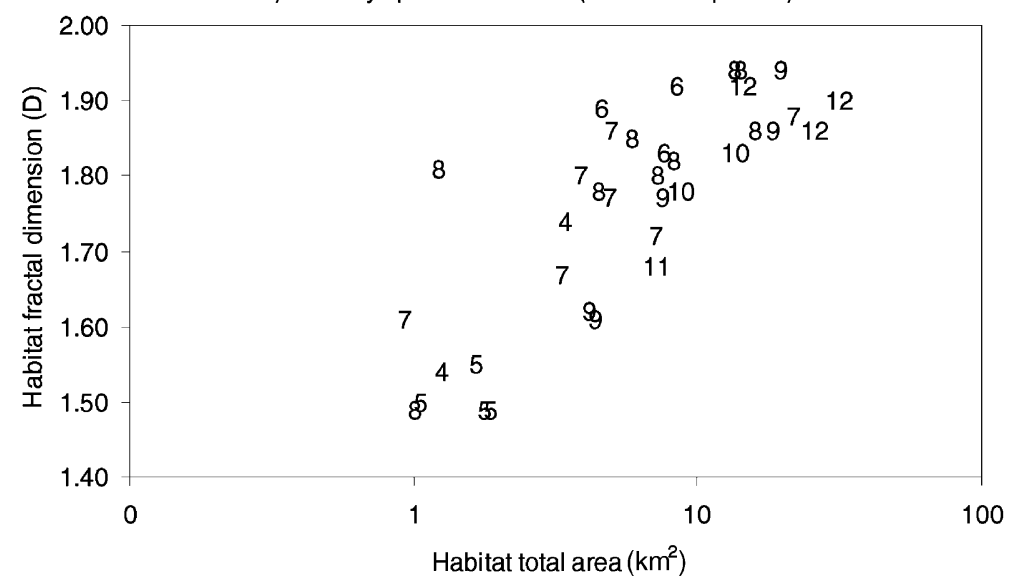

Fig. 8. Illustration of the application of fractal geometry in discriminating between heathland habitat loss (indicated by $h$, proportion heathland habitat) and fractal dimension $(D)$ in nine Dutch landscapes of each $9 \mathrm{~km} \times 9 \mathrm{~km}$ Vertical comparisons are landscapes with the same fractal dimension (indicating habitat contiguity) but with different total cover of habitat, while horizontal comparisons are landscapes with the total amount of habitat, but decreasing contiguity $(D)$.

The proportional fill of the landscape seems to constrain the number of possible combinations of the fractal dimension and lacunarity. When the proportional fill increases to 1 , the fractal dimension approaches 2 (a fully two-dimensional shape) and the lacunarity goes to 0 (no texture). Towards lower and lower proportional fill, the maximum achievable fractal dimensions decreases, as more "edge" habitat is formed, decreasing contiguity.

Although almost 100 different indices of indices of spatial structure in landscapes are currently used in landscape ecology (Riitters et al., 1995), we think that fractal geometry is an especially powerful tool for characterising spatial pattern. This is because it allows the calculation of the amount of something (resource, food, habitat) as function of the spatial scale at which an organism "views" its resources (Fig. 4). Therefore, the spatial features of the pattern (lacunarity, fractal dimension) can be used in resource-based competition models, and consequences for diversity can be calculated (Ritchie and Olff, 1999). With this modelling approach, we can theoretically calculate how many species will be lost within competing assemblages as a result of habitat fragmentation. Case studies to experimentally test the theoretical predictions currently in progress. 


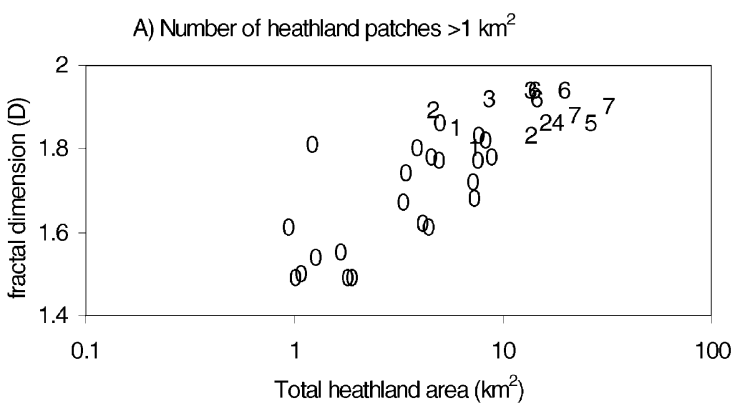

B) Black grouse breeding occurrence

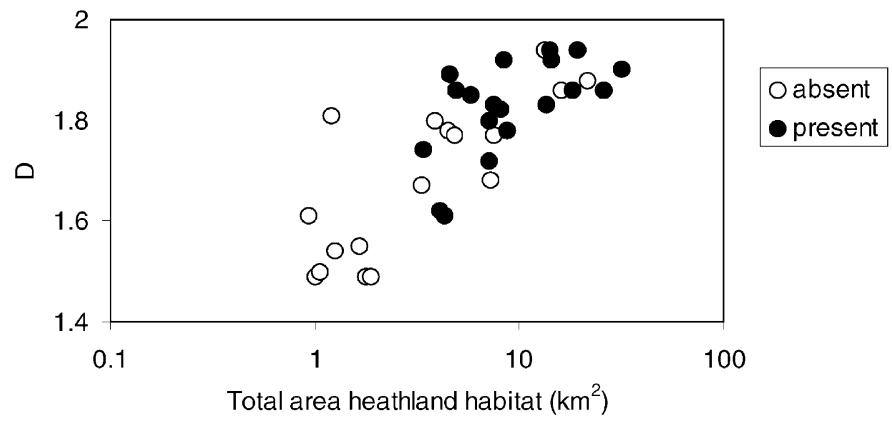

Fig. 9. Effect of habitat loss (proportion heathland habitat) and habitat fragmentation (as indicated by the fractal dimension) on species richness of birds and butterflies in 36 Dutch landscapes of each $9 \mathrm{~km} \times 9 \mathrm{~km}$. Only species characteristic for heathlands were used in the calculation, to account for differences in the remaining ("white") habitat between the landscapes.

\section{Case study: biodiversity in fractal landscapes}

The usefulness of fractal geometry in analysing the response of species richness to landscape fragmentation is currently tested in a descriptive study involving 36 Dutch landscapes of each $9 \mathrm{~km} \times 9 \mathrm{~km}$. In this part, we will report some first results of this study. Within each landscape, the occurrence of heathland habitat was digitised from 1:50.000 scale topographic maps. Furthermore, we could use data on the occurrence of all higher plant species, breeding birds and butterflies, collected in a $1 \mathrm{~km} \times 1 \mathrm{~km}$ grid between 1970 and 1990 in annual surveys by volunteers, with the data collection managed by several NGOs (SOVON for birds, Vlinderstichting for butterflies, and FLORON for plants). We used the aggregated data over time for this period, as not much has changed to the landscape spatial structure over this time period. We calculated the mass fractal dimension according to the method outlined discussed for Fig. 5.

Fig. 7 shows how well the proportional fill (all at the same resolution) and the fractal dimension can be used to separate habitat loss from habitat fragmentation. Note that the fractal dimension $D$, (reflecting habitat contiguity), appears to be a very good measure for fragmentation, and that the methods discriminates fragmentation very nicely from habitat loss. A high value for $D$ indicates that more habitat is encountered quickly when scaling up around a given piece (occupied cell) of habitat. For this dataset, very high lacunarities (indicating fine textures, Fig. 6) did not occur. In fact, lacunarity and proportional fill were positively correlated for the dataset of all landscapes. The consequences of the differences in amount of habitat and fragmentation (as indicated by $D$ are shown for heathland breeding birds and heathland butterflies in Fig. 8.

For breeding birds, these first results indicate that habitat loss and habitat fragmentation both have a negative and independent effect on the number of species that occurs in these landscapes. For butterflies, habitat loss also has an effect, while the effect of fragmentation is less clear. Further analyses of these data are necessary to explain these differences. For butterflies, the resolution of mapping may have been 
too coarse to indicate the relevant fragmentation in each landscape. For birds, this mapping scale may have been more appropriate. Another methodological problem still to be solved is the strong covariance between total habitat area and fractal dimension for the full dataset, making it more difficult to separate their unique effects. For this, analysis of the subsets of landscapes with a better separation may be necessary. Nevertheless, we conclude that fractal geometry seems to be a promising approach enabling the linkage of population and community processes to landscape spatial structure (Fig. 9).

\section{Acknowledgements}

We are thank the Dutch NGOs Vlinderstichting (butterflies), SOVON (breeding birds) and FLORON (plants) and all the volunteers that collected these data for providing data on species richness for the 36 Dutch landscapes we studied. Han Olff was supported by The Netherlands Organisation for Scientific Research (NWO), WOTRO programme Biodiversity in Disturbed Ecosystems. Mark Ritchie was supported by the US National Science Foundation, a travel grant from NWO and by the US Ecology Center.

\section{References}

Brown, J.H., Maurer, B.A., 1989. Macroecology: the division of food and space among species on continents. Science 243, 1150.

Chaneton, E.J., Facelli, J.M., 1991. Disturbance effects on plant community diversity: spatial scales and dominance hierarchies. Vegetatio 93, 143-156.

Chapin III, F.S., Zavaleta, E.S., Eviner, V.T., Naylor, R.L., Vitousek, P.M., Reynold, H.L., Hooper, D.U., Lavorel, S., Sala, O.E., Hobbie, S.E., Mack, M.C., Diaz, S., 1991. Consequences of changing biodiverisity. Nature 405, 234-242.

Elton, C.S., 1958. The Ecology of Invasion by Animals and Plants. Methuen, London.

Hanski, I., Simberloff, D., 1997. The metapopulation approach, its history, conceptual domain and application to conservation. In: Hanski, I., Gilpin, M.E. (Eds.), Metapopulation Biology. Ecology, Genetics and Evolution. Academic Press, San Diego, pp. 5-26.

Hobbs, R.J., Huenneke, L.F., 1992. Disturbance, diversity, and invasion: implications for conservation. Conserv. Biol. 6, 324337.

Holling, C.S., 1992. Cross-scale morphology, geometry, and dynamics of ecosystems. Ecol. Monogr. 62, 447-502.
Huston, M.A., 1994. Biological diversity. The Coexistence of Species on Changing Landscapes. Cambridge University Press, Cambridge.

Huston, M.A., 1999. Local processes and regional patterns: appropriate scales for understanding variation in the diversity of plants and animals. Oikos 86, 393-401.

Johnson, G.D., Tempelman, A., Patil, G.P., 1995. Fractal based methods in ecology: a review for analysis at multiple spatial scales. Coenoses 10, 123-131.

Knapp, A.K., Blair, J.M., Briggs, J.M., Collins, S.L., Hartnett, D.C., Johnson, L.C., Towne, E.G., 1999. The keystone role of bison in north American tallgrass prairie: bison increase habitat heterogeneity and alter a broad array of plant, community, and ecosystem processes. Bioscience 49, 39-50.

MacArthur, R.H., Wilson, E.O., 1967. The Theory of Island Biogeography. Princeton University Press, Princeton.

Mandelbrot, B., 1987. The Fractal Geometry of Nature. Freeman, San Francisco.

Milne, B.T., 1991. The utility of fractal geometry in landscape design. Landscape Ecol. 21, 81-90.

Milne, B.T., 1992. Spatial aggregation and neutral models in fractal landscapes. Am. Nature 139, 32-57.

Milne, B.T., Turner, M.G., Wiens, J.A., Johnson, A.R., 1992. Interactions between the fractal geometry of landscapes and allometric herbivory. Theor. Pop. Biol. 41, 337-353.

O’Neill, R.V., Milne, B.T., Turner, M.G., Gardner, R.H., 1989. Resource utilization scales and landscape pattern. Landscape Ecol. 2, 63-69.

Pärtel, M., Zobel, M., 1996. The species pool and its relation to species richness: evidence from Estonian plant communities. Oikos 75, 111-117.

Ricklefs, R.E., Schluter, D., 1993. Species diversity: regional and historical influences. In: Ricklefs, R.E., Schluter, D. (Eds.), Species Diversity in Ecological Communities. University of Chicago Press, Chicago, pp. 350-363.

Riitters, K.H., O’Neill, R.V., Hunsaker, C.T., Wickham, J.D., Yankee, D.H., Timmins, S.P., Jones, K.B., Jackson, B.L., 1995. A factor analysis of landscape pattern and structure metrics. Landscape Ecol. 10, 23-39.

Ritchie, M.E., Olff, H., 1999. Spatial scaling laws yield a synthetic theory of biodiversity. Nature 400, 557-560.

Rosenzweig, M.L., 1995. Species Diversity in Space and Time. Cambridge University Press, Cambridge.

Schlesinger, W.H., 1991. Biogeochemistry: An Analysis of Global Change. Academic Press, San Diego.

Tilman, D., 1982. Resource Competition and Community Structure. Princeton University Press, Princeton.

Turner, M.G., O’Neill, R.V., Gardner, R.H., Milne, B.T., 1989. Effects of changing spatial scale on the analysis of landscape pattern. Landscape Ecol. 3, 153-162.

Vos, C.C., Opdam, P., 1993. Patterns and processes in a landscape under stress: the study area. In: Vos, C.C., Opdam, P. (Eds.), Landscape Ecology of a Stressed Environment. Chapman \& Hall, London, pp. 1-27.

Wilson, E.O., Willis, E.O., 1975. Applied biogeography. In: Cody, M.L., Diamond, J.M. (Eds.), Ecology and Evolution of Communities. Harvard University Press, Cambridge, MA, pp. 523-534. 